\title{
Subinterlobular Pleural Location Is a Risk Factor for Pneumothorax After Bronchoscopy
}

\author{
Haruka Chino MD, Motoyasu Iikura MD PhD, Nayuta Saito MD, Nahoko Sato MD, \\ Manabu Suzuki MD, Satoru Ishii MD, Eriko Morino MD, Go Naka MD, Jin Takasaki MD, \\ Shinyu Izumi MD PhD, Masayuki Hojo MD PhD, Yuichiro Takeda MD PhD, and \\ Haruhito Sugiyama MD PhD
}

\begin{abstract}
BACKGROUND: Pneumothorax is one of the most important complications after bronchoscopy. This study was conducted to determine the risk factors for post-bronchoscopy pneumothorax. METHODS: We retrospectively reviewed the medical records of 23 consecutive subjects who were diagnosed with iatrogenic pneumothorax after bronchoscopy between August 2010 and February 2014. Forty-six control subjects who did not develop pneumothorax after bronchoscopy were randomly selected. The factors affecting the occurrence of pneumothorax were determined by univariate and multivariate analyses. RESULTS: Among 991 patients who underwent bronchoscopy during the study period, 23 (2.3\%) developed pneumothorax after bronchoscopy. Among these 23 subjects, $13(57 \%)$ required chest tube drainage. Compared with the control group (46 randomly selected from 968 subjects who did not develop pneumothorax), the group that developed pneumothorax had a preponderance of women and had more target lesions located in the subpleural area (odds ratio [OR] 7.8, 95\% CI 0.9-64), especially those that were close to the interlobular pleura (OR 5.1, 95\% CI 1.6-16.1) and the left lung (OR 3.2, 95\% CI 1.1-9.5). Multivariate analysis revealed that a subinterlobular pleural location of a lesion was a risk factor for pneumothorax (OR 4.8, 95\% CI 1.1-20.4). CONCLUSIONS: Pneumothorax occurred significantly more frequently when bronchoscopy was performed for subinterlobular pleural lesions. Close attention and care should be taken during bronchoscopy, especially when target lesions are abutting the interlobular pleura. Key words: bronchoscopy; pneumothorax; biopsy; risk; pleura; adverse effects. [Respir Care 2016;61(12):1664-1670. (ㅇ 2016 Daedalus Enterprises]
\end{abstract}

\section{Introduction}

Bronchoscopy is an effective diagnostic procedure for different respiratory problems. ${ }^{1}$ With the continuing development of technology and improvement of equipment, bronchoscopy is expected to have a broader range of use with more accurate and safer outcomes. ${ }^{2}$ Endobronchial

\footnotetext{
The authors are affiliated with the Department of Respiratory Medicine, National Center for Global Health and Medicine, Tokyo, Japan. Dr Chino is also affiliated with the Department of Respiratory Medicine, University of Tokyo Hospital, Tokyo, Japan.

The authors have disclosed no conflicts of interest.

Haruka Chino presented a version of this paper at the Japan Society for Respiratory Endoscopy meeting, held April 14 and 15, 2014, in Kyoto, Japan.
}

ultrasound (EBUS)-guided transbronchial biopsy has recently grown in popularity and is increasingly being selected over computed tomography (CT)-guided transthoracic needle biopsy as the diagnostic procedure for peripheral nodules.

Although bronchoscopy is generally safe, specific postprocedural risks, such as pneumothorax, hemorrhage, and infection, remain. Pneumothorax is one of the most im-

\footnotetext{
Correspondence: Haruka Chino MD, Department of Respiratory Medicine, National Center for Global Health and Medicine, 1-21-1 Toyama, Shinjuku-ku, Tokyo 162-8655, Japan. Fax: 81-3-3207-1038; Tel.: 81-33202-7181; E-mail: hchino-tky@umin.ac.jp.
}

DOI: $10.4187 /$ respcare.04874 
portant and common complications related to bronchoscopy, with previous papers reporting an incidence of up to 7.5\%.1-6 In clinical practice, some measures may be undertaken to prevent pneumothorax. For example, pneumothorax may be prevented by confirming the biopsy site by fluoroscopy and by checking whether the patient feels pain. However, the latter may not be always feasible, especially in sedated patients.

Given the increasing applicability of bronchoscopy for peripheral lesions and the frequent use of sedatives, the incidence of complications such as pneumothorax is also expected to increase in the future. To date, only a few studies have reported on the risk factors for pneumothorax after bronchoscopy. Specifically, some studies have mentioned that women and patients with COPD or emphysema on high-resolution CT were more likely to develop pneumothorax. ${ }^{7,8}$ However, the relation between the lesion location and the pneumothorax after bronchoscopy remains unclear. The goal of our study was to determine the subject-, lesion-, and procedure-related factors that are associated with the development of pneumothorax after bronchoscopy.

\section{Methods}

We retrospectively reviewed the medical records of consecutive subjects who developed pneumothorax after bronchial brushing or transbronchial biopsy for the diagnosis of peripheral lung lesions between August 2010 and February 2014 at the National Center for Global Health and Medicine (Tokyo, Japan). The clinical characteristics of subjects; location, features, and size ( $\leq 3 \mathrm{~cm}$ vs $>3 \mathrm{~cm}$ ) of target lesions; and the variables related to the bronchoscopy procedure were examined. To identify the risks associated with pneumothorax after bronchoscopy, we compared subjects who developed pneumothorax and those who did not develop pneumothorax after bronchoscopy. Among 968 patients who did not develop pneumothorax after bronchial brushing or transbronchial biopsy during the same period, 46 subjects were randomly selected as the control group using a random number table.

The end point was the association of the study variables with the development of pneumothorax after bronchoscopy. The location of target lesions was classified based on its relationship with the pleura: apical pleura, basal pleura, interlobular pleura, mediastinal pleura, and lateral pleura, as shown in Figure 1. The apical pleura was defined as the rounded superior portion of the pleura. The basal pleura was defined as the portion in contact with the diaphragm. The mediastinal pleura was defined as the portion in contact with the mediastinum. The interlobular pleura was defined as the portion that was sandwiched between 2 lobes. The lateral pleura was the portion other than the apical pleura, basal pleura, interlobular pleura,

\section{QUICK LOOK}

\section{Current knowledge}

With the development of flexible bronchoscopes and biopsy techniques, including endobronchial ultrasound, the smaller lung nodules have been targeted for biopsy by bronchoscopy. Pneumothorax is one of the most important and common complications related to bronchoscopy. Women and patients with COPD or emphysema on high-resolution $\mathrm{CT}$ have been reported to develop pneumothorax more frequently, but the lesionand procedure-related factors that are associated with the development of pneumothorax after bronchoscopy have not been elucidated.

\section{What this paper contributes to our knowledge}

In this retrospective study, target lesions that were located close to the interlobular pleura were 5 times more frequently associated with pneumothorax after bronchoscopy. Chest tube drainage of pneumothorax after bronchoscopy was required in over half of the subjects. In high-risk cases, the bronchoscopist should pay close attention and perform maneuvers to prevent pneumothorax after the procedure by remaining aware of the pleura or interlobular pleura next to the target lesion.

and mediastinal pleura. The protocol of this study was approved by the institutional review board of the National Center for Global Health and Medicine.

\section{Procedure}

Flexible bronchoscopy using conventional (BF 1T260, Olympus, Tokyo, Japan) and thin (BF P260F, Olympus) bronchoscopes was performed via the oral route under sedation by intramuscular hydroxyzine and intravenous midazolam or propofol. Bronchial brushing was carried out using a disposable cytology brush ( $\Phi 1.8 \mathrm{~mm}$; BC-202D-2010, Olympus). Transbronchial biopsy was performed using biopsy forceps ( $\Phi 1.9 \mathrm{~mm}$ [Endo Jaw FB-231D, Olympus], $\Phi 1.8 \mathrm{~mm}$ [KW1811S, PENTAX Medical, Montvale, New Jersey], or $\Phi 1.5 \mathrm{~mm}$ [KW1511S, PENTAX Medical]). We defined conventional biopsy as transbronchial biopsy without using EBUS guidance to distinguish from EBUS-guided transbronchial biopsy. During the procedure, continuous pulse oximetry was routinely used to monitor oxygen saturation and pulse rate. When necessary, oxygen was delivered to the subject via nasal cannula. Blood pressure was intermittently measured.

Secretions in the bronchus were aspirated for cytologic and microbiologic examinations. The lobar bronchus was washed with 1 or $210-\mathrm{mL}$ aliquots of saline. Bronchoal- 


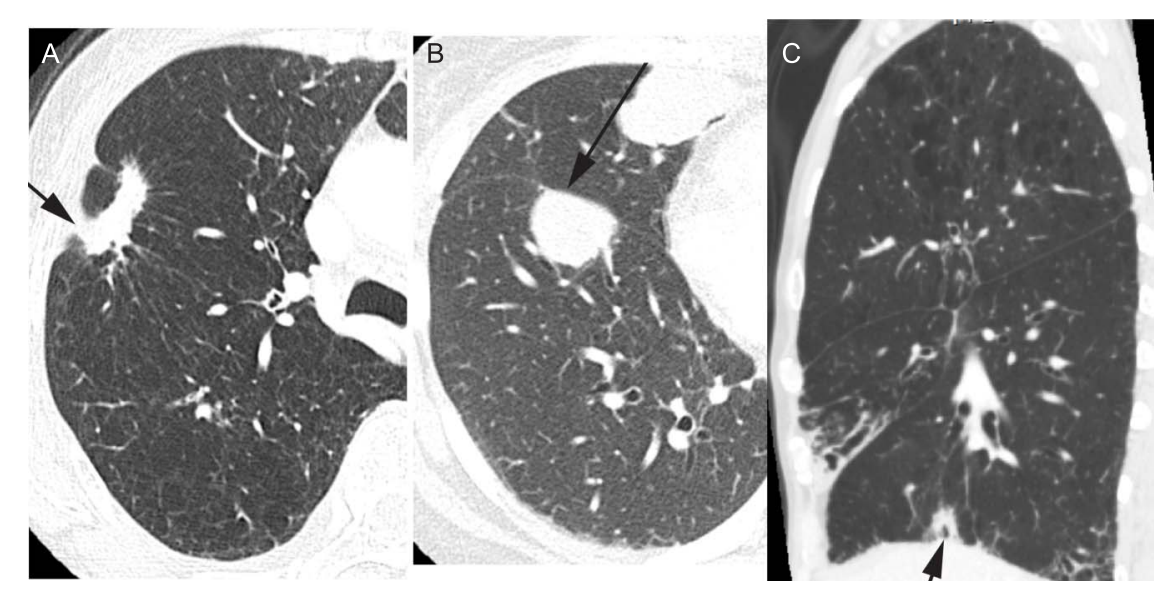

Fig. 1. Examples of the locations of target lesions related to each pleura. A: High-resolution computed tomography shows that the target lesion is located in contact with the lateral pleura (arrow). B: The solid tumor (arrow) is close to the interlobular pleura. C: The small nodule (arrow) is located close to the basal pleura.

veolar lavage was performed by injecting $350-\mathrm{mL}$ aliquots of saline through a bronchoscope that was wedged in the involved segment. Upon insertion of the scope to reach the target bronchus, brush, needle, or forceps with guide sheath was inserted through the working channel and advanced under $\mathrm{x}$-ray fluoroscopic guidance.

All bronchoscopy procedures were performed on an inpatient basis except when there were any reasons that would have prevented hospitalization. The bronchoscopy procedures were performed by pulmonologists under the guidance of an adviser bronchoscopy specialist. After every procedure, the subject was observed for one night before discharge. Chest radiograph was routinely performed several hours after bronchoscopy to detect pneumothorax. We calculated the percentage of pneumothorax by drawing rectangles that demonstrated the outlines of hemithorax and lung and subtracting the respective areas. ${ }^{9,10}$

\section{Statistical Analysis}

Assuming that the percentage for the non-development of pneumothorax after transbronchial biopsy was 40\%, 29 subjects were required for a one-sided type- 1 error rate of 0.05 with $80 \%$ statistical power. Selecting cases and controls in a ratio of 1:2 resulted in a required sample size of 22 for the pneumothorax group.

The association of each outcome with the corresponding set of variables was evaluated using the chi-square test or Wilcoxon-Mann-Whitney test, as appropriate. To identify the risk factors for pneumothorax after bronchoscopy, we used multivariate logistic regression to evaluate the interaction between subjects who developed pneumothorax and those who did not develop pneumothorax. Risk for pneumothorax after bronchoscopy was expressed as the odds ratio (OR). $P$ values of $<.05$ were considered statistically significant, and all tests were 2-sided. We also performed the Hosmer-Lemeshow test to determine the goodness of fit of the logistic regression model. $P$ values of $>.05$ were considered to indicate that the logistic regression model was a good fit. All statistical analyses were performed using SPSS 15.0J for Windows (IBM, Tokyo, Japan).

\section{Results}

\section{Clinical Characteristics and Radiologic Findings of Target Lesions in Subjects Who Developed Pneumothorax After Bronchoscopy}

Among the 991 patients who underwent bronchial washing and/or transbronchial biopsy during the study period, 23 subjects (2.3\%) developed pneumothorax after bronchoscopy. The clinical characteristics of the study population are shown in Table 1. Fourteen subjects (61\%) were women, and the median age was $70 \mathrm{y}$ (range $24-85 \mathrm{y}$ ). Twelve subjects $(52 \%)$ were never-smokers. Five subjects (22\%) were receiving systemic steroids when bronchoscopy was performed. Five subjects had diabetes mellitus.

Emphysema and fibrosis were observed on chest $\mathrm{CT}$ in $6(26 \%)$ and $7(30 \%)$ subjects, respectively. The target lesions were located in the left lung in 10 subjects (43\%) and in the subpleural area in 22 subjects (96\%). Among these 22 subjects, the target lesions were close to the lateral pleura in 13 subjects $(57 \%)$ and to the interlobular pleura in 11 subjects (48\%). Seventeen lesions (74\%) were solid.

\section{Bronchoscopic Procedures Performed}

Transbronchial biopsy was performed for 18 subjects (78\%) (Table 2). In the remaining 5 subjects (22\%), only 
Table 1. Clinical Characteristics and Radiologic Findings of the Target Lesions

\begin{tabular}{|c|c|c|c|}
\hline Characteristics & Pneumothorax $(n=23)$ & Control $(n=46)$ & $P$ \\
\hline Male/female sex, $n(\%)$ & $9 / 14(39 / 61)$ & $30 / 16(65 / 35)$ & .036 \\
\hline Age, median (range) y & $70(24-85)$ & $69.5(38-85)$ & .69 \\
\hline Smoking status, $n(\%)$ & & & .43 \\
\hline Never-smokers & $12(52)$ & $18(39)$ & \\
\hline Ex-smokers & $8(35)$ & $15(33)$ & \\
\hline Current smokers & $3(13)$ & $10(22)$ & \\
\hline Systemic steroid use, $n(\%)$ & $5(22)$ & $3(7)$ & .063 \\
\hline Comorbidity of DM, $n(\%)$ & $5(22)$ & $7(15)$ & .50 \\
\hline Emphysema on CT, $n(\%)$ & $6(26)$ & $12(26)$ & .62 \\
\hline Fibrosis on CT, $n(\%)$ & $7(30)$ & $8(17)$ & .22 \\
\hline Lesion location: left, $n(\%)$ & $10(43)$ & $9(20)$ & .036 \\
\hline Lesion location: upper/middle/lower/diffuse, $n(\%)$ & $6 / 4 / 9 / 4(26 / 17 / 39 / 17)$ & $15 / 5 / 19 / 7(33 / 11 / 41 / 15)$ & .48 \\
\hline Subpleural lesions, $n(\%)$ & $22(96)$ & $34(74)$ & .036 \\
\hline \multicolumn{4}{|l|}{ Portion of pleura, $n(\%)$} \\
\hline Lateral & $13(57)$ & $18(39)$ & .17 \\
\hline Mediastinal & $4(17)$ & $6(13)$ & .14 \\
\hline Interlobular & $11(48)$ & $7(15)$ & .005 \\
\hline Basal & $4(17)$ & $3(7)$ & .16 \\
\hline Apical & $1(4)$ & $6(13)$ & .26 \\
\hline \multicolumn{4}{|l|}{ Character of lesion, $n(\%)$} \\
\hline Solid/Nodular/GGO or consolidation & $17 / 1 / 5(74 / 4 / 22)$ & $31 / 4 / 11(67 / 9 / 24)$ & .77 \\
\hline Diffuse lung disease & $3(13)$ & $7(15)$ & .81 \\
\hline$<30 \mathrm{~mm}$ & $12(52)$ & $14(30)$ & .08 \\
\hline \multicolumn{4}{|l|}{$\begin{array}{l}\mathrm{DM}=\text { diabetes mellitus } \\
\mathrm{CT}=\text { computed tomography } \\
\mathrm{GGO}=\text { ground-glass opacity }\end{array}$} \\
\hline
\end{tabular}

Table 2. Variables Associated With the Occurrence of Pneumothorax After Bronchoscopy

\begin{tabular}{lccc}
\hline \hline \multicolumn{1}{c}{ Variables } & $\begin{array}{c}\text { Pneumothorax } \\
(n=23)\end{array}$ & $\begin{array}{c}\text { Control } \\
(n=46)\end{array}$ & $P$ \\
\hline Lung biopsy, $n(\%)$ & $18(78)$ & $41(89)$ & .20 \\
$\quad$ EBUS-GS & $16(70)$ & $28(61)$ & .33 \\
$\quad$ Within & $13(57)$ & & \\
$\quad$ adjacent to & $1(4)$ & & \\
$\quad$ Outside & $2(9)$ & & \\
$\quad$ Conventional biopsy & $4(17)$ & & .47 \\
Peripheral brushing, $n(\%)$ & $20(87)$ & $38(83)$ & \\
Brushing without biopsy, $n(\%)$ & $5(22)$ & & .005 \\
Thin bronchoscopy, $n(\%)$ & $11(48)$ & $7(15)$ & \\
Dose of midazolam, median & $2.2(1-8)$ & $1.8(0-6.3)$ & \\
$\quad$ (range) mg & $13(57)$ & $16(35)$ & .18 \\
$\begin{array}{l}\text { Dose of midazolam }>2 \text { mg, } \\
\quad n(\%)\end{array}$ & $9(39)$ & $17(37)$ & .53 \\
Treatment with thrombin, $n(\%)$ & $44(20-75)$ & .001 \\
Duration of the procedure, & $57(32-112)$ & & \\
$\quad$ median (range) min & & & \\
\hline EBUS-GS = endobronchial ultrasonography with a guide sheath & & \\
\hline
\end{tabular}

peripheral brushing was performed. In 16 subjects (70\%), radial EBUS with a guide-sheath transbronchial biopsy was performed; EBUS images revealed "within" in 13 subjects and "adjacent to" in one subject. In the remaining 2 subjects, target lesions could not be detected by EBUS. Conventional transbronchial lung biopsy was performed on 4 subjects $(17 \%)$. The median time required for the procedures was $57 \mathrm{~min}$ (range 32-112 $\mathrm{min}$ ). While performing bronchoscopy, administration of thrombin was needed to stop bleeding in 9 subjects (39\%).

\section{Outcome After Pneumothorax}

Definitive diagnosis was confirmed by bronchoscopy in 13 subjects (57\%) (Table 3). Pneumothorax was detected on fluoroscopy immediately after bronchoscopy in 5 subjects $(22 \%)$ and on chest radiograph several hours after bronchoscopy in 18 subjects (78\%). Among the 23 subjects, chest tube drainage was required in 13 subjects (57\%) and pleurodesis in one subject (4\%). Fourteen subjects (61\%) needed supplemental oxygen therapy. The median interval to resolution of pneumothorax was $1 \mathrm{~d}$ (range $0-8 \mathrm{~d}$ ). The median duration until discharge was $5 \mathrm{~d}$ (range 1-72 d). The most frequent final diagnosis was primary 
Table 3. Outcomes After Pneumothorax

\begin{tabular}{lc}
\hline \multicolumn{1}{c}{ Variables } & $\begin{array}{c}\text { Subjects } \\
(n=23)\end{array}$ \\
\hline $\begin{array}{l}\text { Diagnosis achieved, } n(\%) \\
\text { Pneumothorax detected by fluoroscopy, } n(\%)\end{array}$ & $13(57)$ \\
Percentage volume of pneumothorax, & $5(22)$ \\
$\quad$ median (range) $\%$ & $20(1-80)$ \\
Treatment of pneumothorax, $n(\%)$ & \\
$\quad$ Observation & $7(30)$ \\
Supplemental oxygen therapy & $14(61)$ \\
Chest tube drainage & $13(57)$ \\
Pleurodesis & $1(4)$ \\
Time to resolution, median (range) d & $1(0-8)$ \\
Days to resolution, $n$ (\%) & $14(61)$ \\
$0-1$ & $9(39)$ \\
$>2$ & $5(1-72)$ \\
Time to discharge, median (range) d
\end{tabular}

Table 4. The Final Diagnoses of the Target Lesions

\begin{tabular}{llcc}
\hline \hline \multicolumn{1}{c}{ Diagnosis } & $\begin{array}{c}\text { Pneumothorax } \\
(n=23)\end{array}$ & Control $(n=46)$ & $P$ \\
\hline Primary lung cancer & $7(30 \%)$ & $25(54 \%)$ & .060 \\
Metastatic lung cancer & $2(9 \%)$ & $4(9 \%)$ & $>.99$ \\
Diffuse lung disease & $2(9 \%)$ & $6(13 \%)$ & .59 \\
Mycobacterium infection & $3(13 \%)$ & $3(7 \%)$ & .36 \\
Inflammation & $2(9 \%)$ & $7(15 \%)$ & .45 \\
Unknown & $7(30 \%)$ & $1(2 \%)$ & .005 \\
& & & \\
Results are $n(\%)$. & & & \\
\hline
\end{tabular}

lung cancer (30\%), followed by Mycobacterium infection (13\%), as shown in Table 4.

\section{Case Control Study}

The characteristics of the control group are shown in Table 1 . The number of women in the study group was significantly higher than that in the control group $(61 \%$ vs $35 \%, P=.036)$. The proportion of female sex in the control group was similar to that in the overall population (35\% vs $39 \%)$. There were no significant differences between the 2 groups with regard to age; smoking status; comorbidity; underlying lung disease, such as emphysema and fibrosis on high-resolution CT; and use of systemic steroids. Compared with the control group, the subjects who developed pneumothorax had target lesions that were more frequently located in the subpleural area (OR 7.8, 95\% CI 0.9-64), especially those that were close to the interlobular pleura (OR 5.1, 95\% CI 1.6-16.1) and the left lung (OR 3.2, 95\% CI 1.1-9.5). Thin bronchoscopy was more commonly used in the pneumothorax group (Table 2) than the control group. There were no significant dif-
Table 5. Risk Factors Associated With Pneumothorax After Bronchoscopy

\begin{tabular}{|c|c|c|c|c|}
\hline \multirow{2}{*}{ Risk Factor } & \multirow{2}{*}{$\begin{array}{l}\text { Univariate } \\
\text { Analysis, } P\end{array}$} & \multicolumn{3}{|c|}{ Multivariate Analysis } \\
\hline & & Odds Ratio & $95 \% \mathrm{CI}$ & $P$ \\
\hline Thin bronchoscope type & .005 & 3.66 & $0.94-14.1$ & .060 \\
\hline Interlobular pleura & .005 & 4.82 & $1.14-20.4$ & .032 \\
\hline Sampling from the left lung & .040 & 2.48 & $0.6-9.09$ & .19 \\
\hline Male sex & .042 & & & $\mathrm{NE}$ \\
\hline Subpleural lesion & .057 & 4.38 & $0.37-52.1$ & .24 \\
\hline Systemic steroid use & .08 & 3.29 & $0.53-20.4$ & .20 \\
\hline Small size $(\leq 3 \mathrm{~cm})$ & .08 & 1.9 & $0.45-7.69$ & .38 \\
\hline Current + former smoker & .13 & 1.37 & $0.56-3.26$ & .47 \\
\hline \multicolumn{5}{|c|}{$\begin{array}{l}\text { Univariate analysis was performed by the chi-square test. Multivariate analysis was performed } \\
\text { by logistic regression. Hosmer-Lemeshow test: } P=.87 \\
\mathrm{NE}=\text { not evaluated }\end{array}$} \\
\hline
\end{tabular}

ferences between the 2 groups with regard to the procedures (Table 2). With regard to the final diagnosis, significantly more subjects did not receive definite diagnosis in the pneumothorax group (Table 4). However, there was no difference in yield of the etiology of the lesions in the 2 groups, as shown in Table 4. The study group had a significantly longer bronchoscopy procedure time than the control group. Multivariate analysis revealed that subinterlobular pleural location was a risk factor for pneumothorax after bronchoscopy (OR 4.8, 95\% CI 1.1-20.4) (Table 5).

\section{Discussion}

We reported in this study the characteristics and outcomes of subjects who developed pneumothorax after bronchoscopy. We presented in detail the risk factors associated with pneumothorax after bronchoscopy. The present study addressed 3 important clinical issues.

First, target lesions that were located close to the interlobular pleura were 5 times more frequently associated with pneumothorax after bronchoscopy. Generally, subpleural lesions are more likely to develop pneumothorax due to direct damage to the pleura during forceps biopsy and brushing. To the best of our knowledge, there have been no reports about the association between the location in the pleura and pneumothorax after bronchoscopy, although a previous paper reported that there was no difference in the occurrence rate of pneumothorax between lesions in the outer one third of the lung versus the central two thirds of the lung evaluated by CT. ${ }^{4}$ To date, fluoroscopy has been used to localize peripheral lesions and facilitate appropriate bronchoscopy procedures. Fluoroscopy enables confirmation of the positional relationship between the pleura and forceps when a target lesion is close to the lateral pleura, but it does not clearly demonstrate the in- 
terlobular pleura. ${ }^{11,12}$ In addition, it is difficult to confirm damage to the interlobular pleura, regardless of the presence of pain. The interlobular pleura contains 2 visceral pleural surfaces, which have no pain fibers, unlike the parietal pleura ${ }^{13}$; therefore, subjects will not feel pain even when the biopsy forceps has reached the interlobular pleura.

Second, fluoroscopy immediately after bronchoscopy was not able to detect pneumothorax in $78 \%$ of subjects. Routine chest radiograph after bronchoscopy was not recommended by the British Thoracic Society Guidelines because it rarely provides useful clinical information in the absence of symptoms. ${ }^{12}$ In addition, the occurrence of pneumothorax is often delayed for up to $2 \mathrm{~h}$ after bronchoscopy in $40 \%$ of cases. ${ }^{12}$ Pneumothorax has been reported to become visible $1 \mathrm{~h}$ after bronchoscopy in some patients, although it was not detected by chest radiograph immediately after CT-guided transthoracic needle biopsy., ${ }^{2,14-17} \mathrm{In}$ some previous reports, performance of chest radiograph $24 \mathrm{~h}$ after a procedure was required to detect pneumothorax, and monitoring of oxygen saturation was recommended. ${ }^{2}$ Even if fluoroscopy immediately after bronchoscopy does not reveal pneumothorax, chest radiograph examination should be performed $2-4 \mathrm{~h}$ after bronchoscopy if patients complain of breathlessness or if oxygen saturation by pulse oximetry decreases. Regardless of the presence of chest pain during procedures, operators should monitor the respiratory status and conduct chest radiograph examinations as necessary.

Third, chest tube drainage of pneumothorax after bronchoscopy was required in $57 \%$ of our subjects and in $40-$ $70 \%$ of those reported in previous studies. ${ }^{12,18-22}$ According to the British Thoracic Society Guidelines for the management of pneumothorax,,$^{23}$ drainage is needed if patients complain of breathlessness or in patients with $>1 \mathrm{~cm}$ interpleural distance at the hilar level. According to the American College of Chest Physicians Delphi consensus statement, insertion of a chest catheter is recommended in secondary pneumothorax, unless the apex-to-cupola distance is $<3 \mathrm{~cm}$, and in patients who meet all of the following criteria: (1) breathing frequency $<24$ breaths/min; (2) heart rate $>60$ or $<120$ beats/min; (3) normal blood pressure; (4) oxygen saturation $>90 \%$ at room air; and (5) ability to speak in whole sentences in between breaths. ${ }^{24}$ Regardless, pneumothorax after bronchoscopy is iatrogenic; therefore, careful determination of appropriate treatment is necessary.

Our results provided an important clinical implication: Careful attention should be paid during bronchoscopy when target lesions are located close to the interlobular pleura. In the future, diagnostic bronchoscopy for peripheral lung lesions will be increasingly used due to the development of methods such as electromagnetic navigation bronchoscopy and radial EBUS-guided lung biopsy.
To decrease the risk of pneumothorax, it is necessary to confirm the tangential direction of pleura. The location of the biopsy device tip should be confirmed and marked on fluoroscopy during slow insertion to the peripheral part of the lung. Operators should not insert the forceps or brush more distal than this mark. The response of patients to the procedure should be observed by avoiding undue sedation. In our study, the dose of midazolam was not significantly different between the pneumothorax subjects and the control, probably because the dose of intravenous midazolam that we used in our hospital was lower than that recommended by the British Thoracic Society guidelines. ${ }^{12}$

Our study has some limitations. The first was the small sample size and the retrospective, single-institution nature of the study design. As a result, the bronchoscopy techniques and procedures varied among cases. Second, the procedures were performed by different physicians, although specialists were always present to guide the bronchoscopy procedure. Third, although the control group had characteristics similar to those of the study group, the possibility of selection bias could not be ruled out.

\section{Conclusions}

Target lesions located close to the interlobular pleura were 5 times more likely to develop pneumothorax after bronchoscopy. This study strongly indicated the necessity of remaining aware of the location of the lesion to be biopsied. In high-risk cases, the bronchoscopist should pay close attention and perform maneuvers to prevent pneumothorax after the procedure.

\section{REFERENCES}

1. Fazlalizadeh H, Adimi P, Kiani A, Malekmohammad M, Jabardarjani HR, Soltaninejad F, et al. Evaluation of bronchoscopy complications in a tertiary health care center. Tanaffos 2014;13(4):48-50.

2. Boskovic T, Stojanovic M, Stanic J, Pena Karan S, Vujasinovic G, Dragisic D, et al. Pneumothorax after transbronchial needle biopsy. J Thorac Dis 2014;6(Suppl 4):S427-S434.

3. Huang Y, Huang H, Li Q, Browning RF, Parrish S, Turner JF Jr, et al. Transbronchial lung biopsy and pneumothorax. J Thorac Dis 2014;6(Suppl 4):S443-S447.

4. Ost DE, Ernst A, Lei X, Kovitz KL, Benzaquen S, Diaz-Mendoza J, et al. Diagnostic yield and complications of bronchoscopy for peripheral lung lesions: results of the AQuIRE registry. Am J Respir Crit Care Med 2016;193(1):68-77.

5. Hayama M, Izumo T, Matsumoto Y, Chavez C, Tsuchida T, Sasada S. Complications with endobronchial ultrasound with a guide sheath for the diagnosis of peripheral pulmonary lesions. Respiration 2015; 90(2):129-135.

6. Gex G, Pralong JA, Combescure C, Seijo L, Rochat T, Soccal PM. Diagnostic yield and safety of electromagnetic navigation bronchoscopy for lung nodules: a systematic review and meta-analysis. Respiration 2014;87(2):165-176.

7. Huang CT, Ruan SY, Liao WY, Kuo YW, Lin CY, Tsai YJ, et al. Risk factors of pneumothorax after endobronchial ultrasound-guided 


\section{Risk Factors For Post-Bronchoscopy PneumothoraX}

transbronchial biopsy for peripheral lung lesions. PLoS One 2012; 7(11):e49125.

8. Tukey MH, Wiener RS. Population-based estimates of transbronchial lung biopsy utilization and complications. Respir Med 2012; 106(11):1559-1565

9. Do S, Salvaggio K, Gupta S, Kalra M, Ali NU, Pien H. Automated quantification of pneumothorax in CT. Comput Math Methods Med 2012;2012;736320.

10. Kircher LT Jr, Swartzel RL. Spontaneous pneumothorax and its treatment. J Am Med Assoc 1954;155(1):24-29.

11. Shulimzon TR. Flexible bronchoscopy in Israel 2010: evidence-based clinical practice guidelines for the adult patient: a concise summary of the recommendations of the Israel Lung Association Task Force. Isr Med Assoc J 2010;12(2):69-73.

12. Du Rand IA, Blaikley J, Booton R, Chaudhuri N, Gupta V, Khalid $\mathrm{S}$, et al. British Thoracic Society guideline for diagnostic flexible bronchoscopy in adults: accredited by NICE. Thorax 2013;68(Suppl 1):i1-i44.

13. Light RW. Pleural diseases, 5th edition. Philadelphia, Pennsylvania: Lippincott Williams \& Wilkins; 2007:73.

14. Charig MJ, Phillips AJ. CT-guided cutting needle biopsy of lung lesions-safety and efficacy of an out-patient service. Clin Radiol 2000;55(12):964-969.

15. Brown KT, Brody LA, Getrajdman GI, Napp TE. Outpatient treatment of iatrogenic pneumothorax after needle biopsy. Radiology 1997;205(1):249-252

16. Traill ZC, Gleeson FV. Delayed pneumothorax after CT-guided percutaneous fine needle aspiration lung biopsy. Thorax 1997;52(6): 581-582; discussion 575-586.
17. Sun SW, Zabaneh RN, Carrey Z. Incidence of pneumothorax after fiberoptic bronchoscopy (fob) in community-based hospital; are routine post-procedure chest roentgenograms necessary? Chest 2003 doi: 10.1378/chest.124.4_MeetingAbstracts.145S-a.

18. Mahajan AK, Patel S, Hogarth DK, Wightman R. Electromagnetic navigational bronchoscopy: an effective and safe approach to diagnose peripheral lung lesions unreachable by conventional bronchoscopy in high-risk patients. J Bronchology Interv Pulmonol 2011; 18(2):133-137.

19. Pue CA, Pacht ER. Complications of fiberoptic bronchoscopy at a university hospital. Chest 1995;107(2):430-432.

20. Colt HG, Matsuo T. Hospital charges attributable to bronchoscopy-related complications in outpatients. Respiration 2001;68(1): 67-72.

21. Milman N, Faurschou P, Munch EP, Grode G. Transbronchial lung biopsy through the fibre optic bronchoscope. Results and complications in 452 examinations. Respir Med 1994;88(10):749753.

22. Ahmad M, Livingston DR, Golish JA, Mehta AC, Wiedemann HP. The safety of outpatient transbronchial biopsy. Chest 1986;90(3): 403-405.

23. MacDuff A, Arnold A, Harvey J. Management of spontaneous pneumothorax: British Thoracic Society Pleural Disease Guideline 2010. Thorax 2010;65(Suppl 2):ii18-ii31.

24. Baumann MH, Strange C, Heffner JE, Light R, Kirby TJ, Klein J, et al. Management of spontaneous pneumothorax: an American College of Chest Physicians Delphi consensus statement. Chest 2001; 119(2):590-602. 